\title{
Efficacy of Preoperative Chemotherapy in Treatment of Children With Wilms' Tumor: A Meta-Analysis
}

\author{
Jia Junjun ${ }^{1}$; Zhou xuelian ${ }^{3}$; Kadel Dhruba ${ }^{2}$; Xie Haiyang ${ }^{1}$; Zhou Lin ${ }^{1}$; Zheng Shusen ${ }^{1, *}$ \\ ${ }^{1}$ Department of Hepatobiliary and Pancreatic Surgery, Key Laboratory of Combined Multi-Organ Transplantation, School of Medicine, First Affiliated Hospital, Zhejiang University, \\ Hangzhou, China \\ ${ }^{2}$ School of Medicine, Zhejiang University, Hangzhou, China \\ ${ }^{3}$ School of Medicine, Zhejiang University, Hangzhou, China
School of Medicine, Children's Hospital, Zhejiang University, Hangzhou, China \\ *Corresponding author: Zheng Shusen, Department of Hepatobiliary and Pancreatic Surgery, Key Laboratory of Combined Multi-Organ Transplantation, School of Medicine, First \\ Affiliated Hospital, Zhejiang University, Hangzhou, China.Tel:+86-57187236567, E-mail: shusenzheng@zju.edu.cn
}

Received: February 20, 2015; Revised: March 6, 2015; Accepted: March 15, 2015

\begin{abstract}
Context: To assess the efficacy of preoperative chemotherapy in Wilms' tumor patients and explore its true value for specific subgroups. Objectives: In the presence of these controversies, a meta-analysis that examines the efficacy of preoperative chemotherapy in Wilms' tumor patients and specific subgroups is needed to clarify these issues. The objective of this meta-analysis is to assess the efficacy of preoperative chemotherapy in Wilms' tumor patients and explore its true value for specific subgroups.

Data Sources: Computer-based systematic search with "preoperative chemotherapy", "Neoadjuvant Therapy" and "Wilms' tumor" as search terms till january 2013 was performed.

Study Selection: No language restrictions were applied.Searches were limited to randomized clinical trials (RCTs) or retrospective studies in human participants under 18 years. A manual examination of references in selected articles was also performed.

Data Extraction: Relative Risk (RR) and their 95\% Confidence Interval (CI) for Tumor Shrinkage (TS), total Tumor Resection (TR), EventFree Survival (EFS) and details of subgroup analysis were extracted. Meta-analysis was carried out with the help of the software STATA 11.0. Finally, four original Randomized Clinical Trials (RCTs) and 28 retrospective studies with 2375 patients were included.

Results: For preoperative chemotherapy vs. up-front surgery (PC vs. SU) group, the pooled RR was 9.109 for TS (95\% CI: 5.109 - 16.241; P < 0.001 ), 1.291 for TR (95\% CI: 1.124 - 1.483; P< 0.001) and 1.101 for EFS (95\% CI: 0.980 - 1.238; P = 0.106). For subgroup short course vs. long course (SC vs. LC), the pooled RR was 1.097 for TS (95\% CI: $0.784-1.563 ; \mathrm{P}=0.587), 1.197$ for TR (95\% CI: $0.960-1.493 ; \mathrm{P}=0.110$ ) and 1.006 for EFS (95\% CI: $0.910-1.250 ; \mathrm{P}=0.430)$.

Conclusions:Short course preoperative chemotherapy is as effective as long course and preoperative chemotherapy only benefits Wilms' tumor patients in tumor shrinkage and resection but not event-free survival.
\end{abstract}

Keywords: Wilms’ Tumor; Event Free Survival; Meta-Analysis

\section{Context}

Wilms' tumor, also known as nephroblastoma, is one of the most common solid tumors in children, the morbidity is 7.8 per million $(1,2)$. The cure rate attained in children with Wilms' tumor has been dramatically improved during the last three decades because of the multidisciplinary approach of combined surgery and multi-drug therapy, and use of radiotherapy (RTH) when necessary. The latest report showed the five-year event-free survival (EFS) and overall survival were $77.2 \%$ and $87.5 \%$ (3). Though there are large leading international organizations focusing on the Wilms' tumor such as SIOP (the International Society of Pediatric Oncology) and NWTSG (the National Wilms' Tumor Study Group), they have different opinions regarding the administration of preoperative chemotherapy. The NWTSG advocates up-front resection of the primary tumor before chemotherapy is given. In contrast, SIOP recommends the administration of chemotherapy before surgery. What's more, the short course before surgery was proposed to further reduce the toxicity but the efficacy was still debated $(4,5)$.

\section{Objectives}

In the presence of these controversies, a meta-analysis that examines the efficacy of preoperative chemotherapy in Wilms' tumor patients and specific subgroups is needed to clarify these issues. The objective of this meta-analysis is to assess the efficacy of preoperative chemotherapy in Wilms' tumor patients and explore its true value for specific subgroups.

\section{Data Sources}

The aim of this meta-analysis was to collect all available data from clinical trials of preoperative chemotherapy in the treatment of Wilms' tumor. A computer-based systematic search in PubMed (MEDLINE), Cochrane, OVID

Copyright (C) 2015, Growth \& Development Research Center. This is an open-access article distributed under the terms of the Creative Commons Attribution-NonCommercial 4.0 International License (http://creativecommons.org/licenses/by-nc/4.0/) which permits copy and redistribute the material just in noncommercial usages, provided the original work is properly cited. 
(including EBMASE and biosis previews database), VIP, CNKI and WanFang databases before January 2013 with "preoperative chemotherapy", "Neoadjuvant Therapy" and "Wilms' tumor" as search terms was performed for possible clinical trials.

\section{Study Selection}

No language restrictions were applied. Searches were limited to randomized clinical trials (RCTs) or retrospective studies in human participants under 18 years. A manual examination of references in selected articles was also performed. Studies were eligible for inclusion in this meta-analysis if they met the following criteria: I) the design of the studies was RCTs or retrospective studies, II) the preoperative chemotherapy (PC) should have been contained in the research group, whereas up-front surgery without preoperative chemotherapy (SU) in the control group. Or in subgroups, short course (SC, between 2 - 4 weeks) of preoperative chemotherapy has been contained in research group, while long course (LC, more than 4 weeks) in the control group, III) at least one of these endpoint indexes indicating that Tumor Shrinkage (TS), total Tumor Resection (TR) and Event-Free Survival (EFS) were available in the study, IV) the patients should have suffered from Wilms' tumor. Duplicated Reports and Case Reports Were Excluded.

\section{Data Extraction}

\subsection{Quality Assessment}

The quality of selected RCTs was assessed according to the criteria presented in the Cochrane Handbook for Systematic Reviews of Interventions (version 5.0; Chapter 8) (6). Details include random, allocation concealment, Intension-to-Treat (ITT), drop-out and bias risk.

Study characteristics, patient details, total number of patients in each trial, Relative Risk (RR) and their 95\% Confidence Intervals (CI) for Tumor Shrinkage (TS), total Tumor Resection (TR) and Event-Free Survival (EFS) were extracted. We also contacted authors of some undergoing studies for data updates and statistical details. TS was defined as at least a 30\% decrease in the sum of the longest diameter of target lesions with radiological assessment according to the Response Evaluation Criteria in Solid Tumors (RECIST) (7), TR was defined as no residual tumor with naked eyes according to the improved Shamberge criterion, and EFS was defined as no evidence of recurrence and metastasis during two-year follow up.

\subsection{Statistical Analysis}

For studies that provided an effect estimate such as Relative Risk (RR), the study-provided effect estimate was directly used in the pooled meta-analysis calculations. For studies that published number of events but did not provide an effect estimate, this information was used to calculate the RR of each outcome for the intervention compared with the placebo group. We logarithmically transformed the RR and corresponding standard error to stabilize the variance and normalize the distribution. We calculated the overall pooled-effect estimates using inverse-variance weighting to calculate fixed-effects models (8). The Q test was used to assess the presence of heterogeneity and the I-squared index to quantify the extent of heterogeneity (9). To assess for publication bias, we constructed funnel plots for each outcome in which the ln (RR) was plotted against its standard error. The Begg's rank correlation test was used to examine the asymmetry of the funnel plot (10). All analyses were conducted in STATA version 11.0.

\section{Results}

Our search strategies led to collect 724 articles from the published reports. After reviewing each publication, we identified four original RCTs and 28 retrospective studies (among which 13 studies were written in Chinese) with 2375 patients that met our criteria (Figure 1). There are few RCTs about the preoperative chemotherapy of Wilms' tumor, even less about the short course chemotherapy. Meanwhile, several retrospective studies presented meaningful results about these issues; thus, discarding these data would be unwise. The study, intervention, population, quality assessment of RCTs is summarized in Table 1.

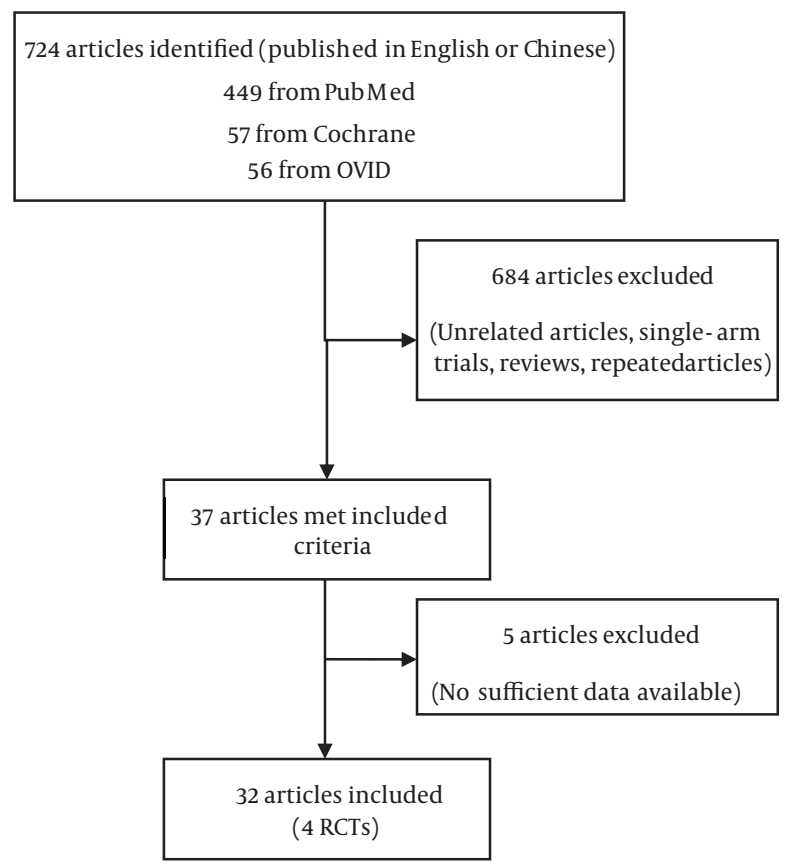

Figure 1. Flow Chart of Study Identification, Rejection and Selection in the Meta-Analysis 
Junjun J et al.

\begin{tabular}{|c|c|c|c|c|c|c|c|}
\hline Author & No. & Intervention & Random & Concealment & ITT & Drop Out & Bias Risk \\
\hline Liu et al. (11) ${ }^{\mathrm{a}}$ & $31 / 26$ & SC vs. LC & - & - & - & - & - \\
\hline Hang et al. (12) ${ }^{\mathrm{a}}$ & $23 / 18$ & PC vs. SU & - & - & - & - & - \\
\hline Sun and $Y i(13)^{a}$ & $10 / 13$ & PC vs. SU & - & - & - & - & - \\
\hline Lu et al. (14) ${ }^{a}$ & $19 / 19$ & PC vs. SU & - & - & - & - & - \\
\hline Gao and Chen (15) ${ }^{\mathrm{a}}$ & $7 / 10$ & PC vs. SU & - & - & - & - & - \\
\hline Huang et al. (16) ${ }^{a}$ & $13 / 6$ & PC vs. SU & - & - & - & - & - \\
\hline Li and Guo $(17)^{a}$ & $17 / 23$ & PC vs. SU & - & - & - & - & - \\
\hline He et al. (4) ${ }^{a}$ & $36 / 26$ & SC vs. LC & - & - & - & - & - \\
\hline Tang et al. (18) ${ }^{\mathrm{a}}$ & $31 / 26$ & SC vs. LC & - & - & - & - & - \\
\hline Li et al. (19) ${ }^{a}$ & $45 / 21$ & SC vs. LC & - & - & - & - & - \\
\hline Zhao et al. (20) ${ }^{\mathrm{a}}$ & $27 / 36$ & PC vs. SU & - & - & - & - & - \\
\hline Tang and $\mathrm{Li}(21)^{\mathrm{a}}$ & $26 / 19$ & PC vs. SU & - & - & - & - & - \\
\hline Li et al. (22) ${ }^{\mathrm{a}}$ & $31 / 20$ & TC VS IC & - & - & - & - & - \\
\hline Naguib et al. (23) ${ }^{\mathrm{b}}$ & $27 / 26$ & PC vs. SU & - & - & - & - & - \\
\hline Yildiz et al. (24) ${ }^{b}$ & $8 / 98$ & PC vs. SU & - & - & - & - & - \\
\hline Sarhan et al. (25) ${ }^{b}$ & $16 / 6$ & PC vs. SU & - & - & - & - & - \\
\hline Sultan et al. (26) ${ }^{b}$ & $19 / 17$ & PC vs. SU & - & - & - & - & - \\
\hline Bogaert et al. (27) ${ }^{b}$ & $17 / 15$ & PC vs. SU & - & - & - & - & - \\
\hline Blakely et al.(28) ${ }^{b}$ & $12 / 8$ & PC vs. SU & - & - & - & - & - \\
\hline Cristofani et al. (29) ${ }^{b}$ & $16 / 6$ & PC vs. SU & - & - & - & - & - \\
\hline Mushtaq et al. (30) ${ }^{\mathrm{b}}$ & $21 / 9$ & PC vs.SU & - & - & - & - & - \\
\hline Kumar et al. (31) ${ }^{\mathrm{b}}$ & $57 / 13$ & PC vs. SU & - & - & - & - & - \\
\hline Maes et al. (32) b & $7 / 8$ & SC vs. LC & - & - & - & - & - \\
\hline Shaul et al. (33) ${ }^{\mathrm{b}}$ & $7 / 8$ & PC vs. SU & - & - & - & - & - \\
\hline Weirich et al. (34) ${ }^{b}$ & $258 / 71$ & PC vs. SU & - & - & - & - & - \\
\hline Coppes et al. (35) ${ }^{\mathrm{b}}$ & $84 / 143$ & PC vs. SU & - & - & - & - & - \\
\hline Guarda et al. (36) ${ }^{b}$ & $21 / 21$ & PC vs. SU & - & - & - & - & - \\
\hline Safdar et al. (37) ${ }^{\mathrm{b}}$ & $21 / 20$ & PC vs. SU & - & - & - & - & - \\
\hline Tournade et al. (5) ${ }^{b}$ & 193/199 & SC vs. LC & yes & yes & yes & no & low \\
\hline Lemerle et al. (38) ${ }^{\mathrm{b}}$ & $73 / 63$ & PC vs. SU & yes & yes & yes & no & low \\
\hline Mitchell et al. (39) ${ }^{b}$ & $92 / 94$ & PC vs. SU & yes & yes & yes & no & low \\
\hline D'Angio et al. $(40)^{b}$ & $13 / 13$ & PC vs. SU & yes & yes & yes & no & low \\
\hline
\end{tabular}

a Retrospective study (Chinese).

b Retrospective study.

6.1. Effect of Preoperative Chemotherapy on Tumor Shrinkage (TS), Tumor Resection (TR), Event-Free Survival (EFS) (Preoperative Chemotherapy vs. UpFront Surgery (PC vs. SU))

Based on the intention-to-treat population, the efficacy of preoperative chemotherapy demonstrated advantages in TS, TR but not EFS.

1) The pooled RR for TS in the 12 studies ( 473 vs. 260) performed by our analysis was 9.109 (95\% CI: 5.109 - 16.241; $\left.\mathrm{P}<0.001 ; \mathrm{I}^{2}=0 \%\right)$, representing a 9.109 times chance of tumor shrinkage in patients with preoperative chemo- therapy (Figure 2). The Begg's test revealed no publication bias.

2) Regarding TR, the pooled RR of 18 studies (394 vs. 317) was 1.291 (95\% CI: 1.124 - 1.483; P $<0.001 ; \mathrm{I}^{2}=22 \%$ ), indicating a 1.291 times chance of total tumor resection in patients with preoperative chemotherapy (Figure 3). The Begg's test revealed no publication bias.

3) The pooled RR for EFS from the 18 studies (547 vs. 589) was 1.101 (95\% CI: 0.980 -1.238; $\mathrm{P}=0.106 ; \mathrm{I}^{2}=0 \%$ ), indicating no statistical difference in tumor recurrence or metastasis after surgery between PC and SU group (Figure 4). The Begg's test also showed no publication bias. 


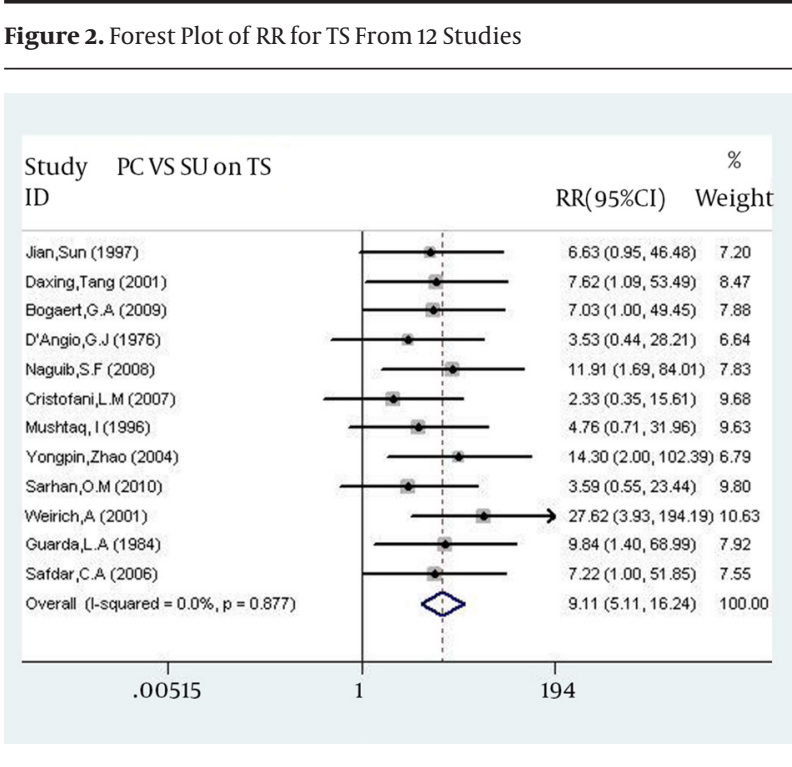

Compared with SU group, preoperative chemotherapy increases the chance of tumor shrinkage before surgery $(R R=9.109, P<0.001)$. It in cludes combined RR calculated by using general inverse variance fixed effects as well as the evaluation for heterogeneity $\left(\mathrm{I}^{2}\right)$. Horizontal lines represent $95 \%$ confidence interval $(95 \% \mathrm{CI}$ ). Boxes indicate the RR point estimate, and their areas are proportional to the weight of the studies. The blue diamond represents the summary estimate. The unbroken vertical line is at the null value $(\mathrm{RR}=1.0)$.

Figure 3. Forest Plot of RR for TR From 18 Studies

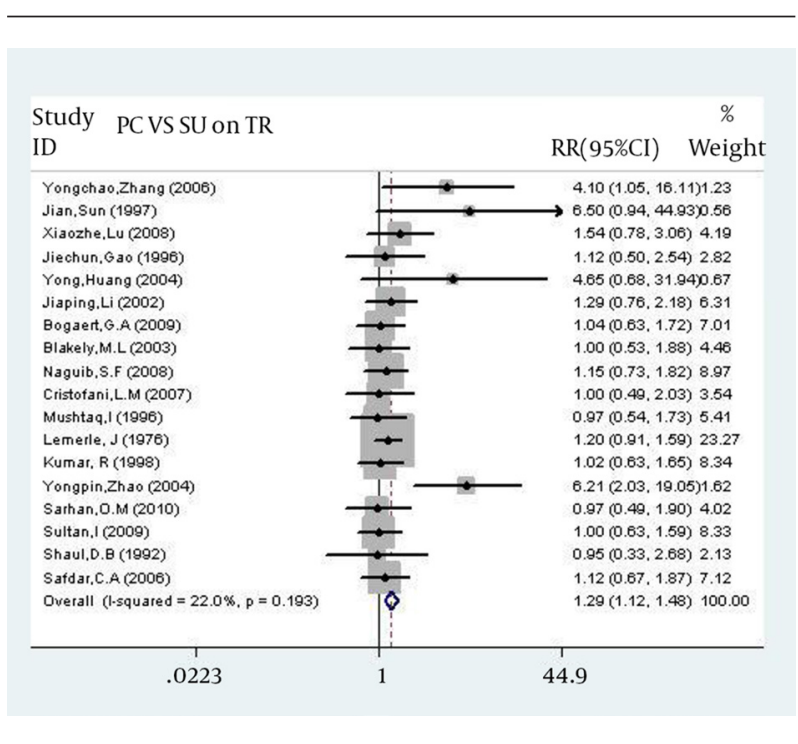

Compared with SU group, preoperative chemotherapy increase the chance of tumor resection ( $R R=1.291, P<0.001)$. It includes combined $R R$ calculated by using general inverse variance fixed effects as well as the evaluation for heterogeneity $\left(\mathrm{I}^{2}\right)$. Horizontal lines represent $95 \%$ confidence interval ( $95 \% \mathrm{CI})$. Boxes indicate the RR point estimate, and their areas are proportional to the weight of the studies. The blue diamond represents the summary estimate. The unbroken vertical line is at the null value $(\mathrm{RR}=1.0)$.
Figure 4. Forest Plot of RR for EFS From 18 Studies

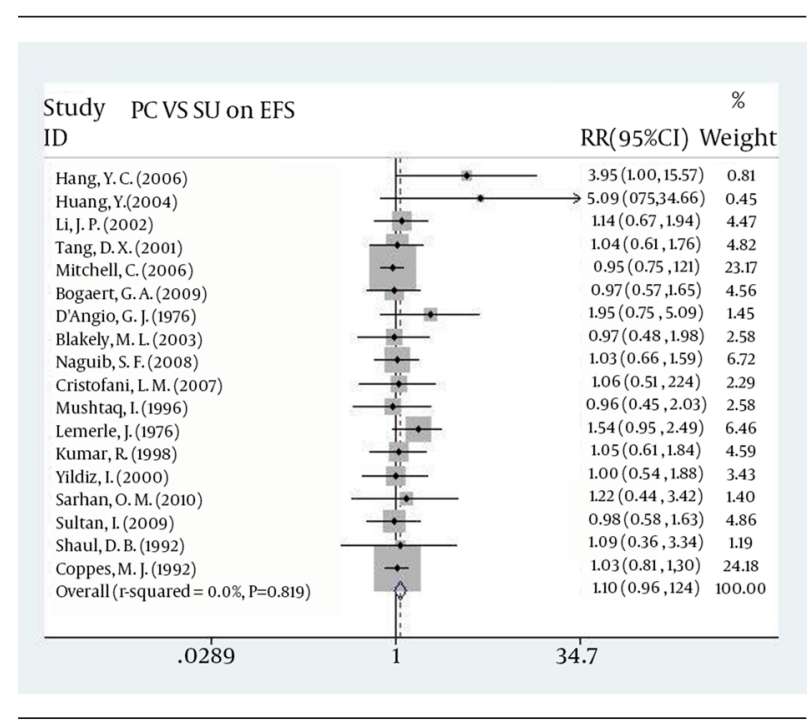

Compared with SU group, preoperative chemotherapy does not reduce Wilms' tumor recurrence or metastasis after surgery $(R R=1.101, P=0.106)$. It includes combined RR calculated by using general inverse variance fixed effects as well as the evaluation for heterogeneity $\left(\mathrm{I}^{2}\right)$. Horizontal lines represent $95 \%$ confidence interval $(95 \% \mathrm{CI}$ ). Boxes indicate the RR point estimate, and their areas are proportional to the weight of the studies. The blue diamond represents the summary estimate. The unbroken vertical line is at the null value $(\mathrm{RR}=1.0)$.

6.2. Subgroup Analysis for Effect of Preoperative Chemotherapy Between Short Course (SC) and Long Course (LC) Group on Tumor Shrinkage (TS), Tumor Resection (TR), Event-Free Survival (EFS)

Based on intention-to-treat population, there were no statistically significant differences between SC and LC group on TS, TR or EFS.

1) The pooled RR for TS in the four studies (76 vs. 63) performed by our analysis was 1.097 (95\% CI: 0.784 - 1.563; P $\left.=0.587 ; \mathrm{I}^{2}=0 \%\right)$, representing the equal chance of tumor shrinkage in patients between SC or LC group (Figure 5). The Begg's test revealed no publication bias.

2) Regarding TR, the pooled RR 5 studies (136 vs.107) was 1.197 (95\% CI: $0.960-1.493 ; \mathrm{P}=0.110 ; \mathrm{I}^{2}=0 \%$ ), indicating no statistical difference in chance of total tumor resection in patients between SC and LC group (Figure 6). The Begg's test revealed no publication bias.

3) The pooled RR for EFS from the two studies (219 vs. 219) was 1.006 (95\% CI: $0.910-1.250 ; \mathrm{P}=0.430 ; \mathrm{I}^{2}=0 \%$ ), indicating no statistical difference in tumor recurrence or metastasis in patients between SC and LC group (Figure 7). The Begg's test revealed no publication bias.

\section{Conclusions}

The progress in treatment of Wilms' tumor is one of the greatestachievementsinoncologyforthelastthreedecades. 


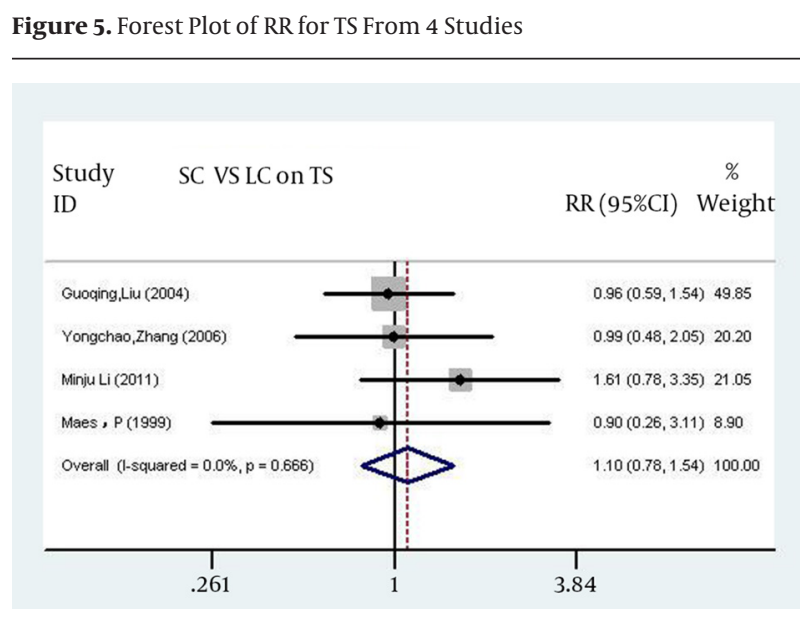

Compared with LC group, short course of preoperative chemotherapy is as effective as long course in TS ( $R R=0.097, \mathrm{P}=0.587)$. It includes combined RR calculated by using general inverse variance fixed effects as well as the evaluation for heterogeneity $\left(\mathrm{I}^{2}\right)$. Horizontal lines represent $95 \%$ confidence interval (95\% CI). Boxes indicate the RR point estimate, and their areas are proportional to the weight of the studies. The blue diamond represents the summary estimate. The unbroken vertical line is at the null value $(\mathrm{RR}=1.0)$.

Modern treatment regimens yield overall survival rates of $87.5 \%$, and this success has precipitated a shift in emphasis on reducing treatment related toxicity. Although North America and Europe have different philosophies on preoperative chemotherapy, both treatment approaches yield excellent clinical outcomes (41). The SIOP strategy of giving preoperative chemotherapy is based on the premise that it usually reduces the tumor volume, thereby decreasing the likelihood of spillage, "downstaging" the tumor and improving the tumor resection (42). Our meta-analysis focuses on this premise and related indexes, the pooled RR for TS, TR in our meta-analysis are 9.109 (95\% CI: $5.109-16.241 ; \mathrm{P}<0.001 ; \mathrm{I}^{2}=0 \%$ ) and 1.291 (95\% CI: $1.124-1.483 ; \mathrm{P}<0.001 ; \mathrm{I}^{2}=22 \%$ ), representing that the chance of tumor shrinkage is nine times higher and the chance of total tumor resection is 1.3 times higher in patients with preoperative chemotherapy compared to the patients with up-front surgeries. On the premise of SIOP previous data and its treatment regimen, this study yields slightly variable outcomes in the prospective of preoperative chemotherapy in the patients with Wilms' tumor, as the pooled RR for EFS in this study is 1.101 (95\% CI: $0.980-1.238 ; \mathrm{P}=0.106 ; \mathrm{I}^{2}=0 \%$, indicating no statistical difference in the case of Wilms' tumor recurrence or metastasis after surgery in the patient with preoperative chemotherapy and up-front surgery, which concludes that whether preoperative chemotherapy is given or not, both approaches yield same clinical outcomes after may provide a valuable prognostic indicator (43). Some problems also should be noticed, the most relevant one is reported by SIOP 93 - 01 that approximately 5\% of lesions in patients treated with preoperative chemotherapy
Figure 6. Forest Plot of RR for TR From 5 Studies

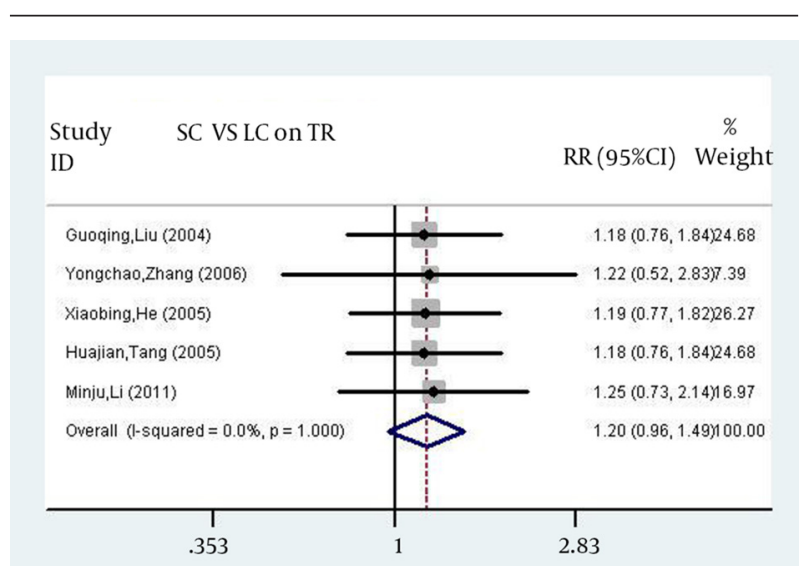

Compared with LC group, short course of preoperative chemotherapy is as effective as long course in $T R(R R=1.197, P=0.110)$. It includes combined RR calculated by using general inverse variance fixed effects as well as the evaluation for heterogeneity $\left(\mathrm{I}^{2}\right)$. Horizontal lines represent 95\% confidence interval $(95 \% \mathrm{CI})$. Boxes indicate the RR point estimate, and their areas are proportional to the weight of the studies. The blue diamond represents the summary estimate. The unbroken vertical line is at the null value $(R R=1.0)$.

Figure 7. Forest Plot of RR for EFS From 2 Studies

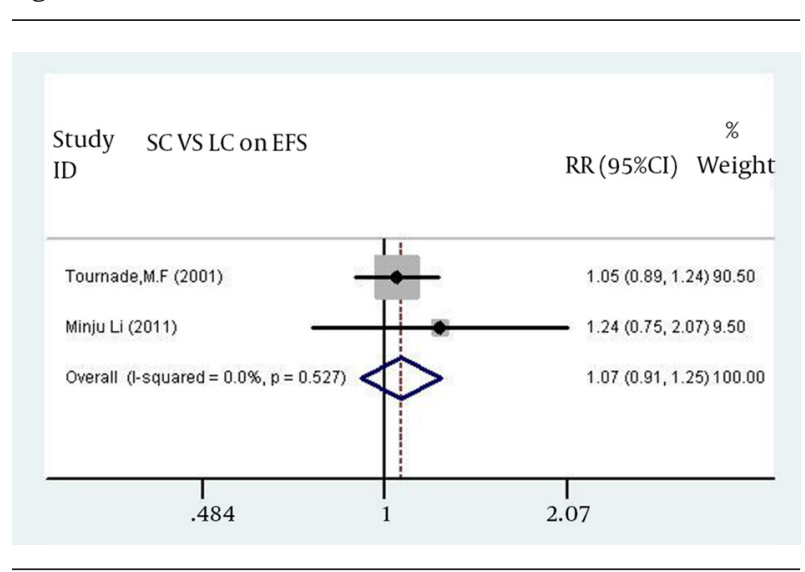

Compared with LC group, short course of preoperative chemotherapy is as effective as long course in tumor recurrence or metastasis after surgery $(R R=1.006, P=0.430)$. It includes combined $R R$ calculated by using general inverse variance fixed effects as well as the evaluation for heterogeneity $\left(\mathrm{I}^{2}\right)$. Horizontal lines represent $95 \%$ confidence interval (95\% CI). Boxes indicate the RR point estimate, and their areas are proportional to the weight of the studies. The blue diamond represents the summary estimate. The unbroken vertical line is at the null value $(\mathrm{RR}=1.0)$.

were ultimately shown not to be Wilms' tumor and included $1.8 \%$ benign tumors (44); there is considerable hematological toxicity and treatment-related mortalityin malnourished children, for whom reduced doses should be considered (45). The other important consequence is, certain chemotherapeutic agents such as doxorubicin, dactinomycin, and vincristine may contribute to an increased risk of secondary malignancies. Fifteen years 
after initial diagnosis, the cumulative incidence of a secondary malignant neoplasm in patients registered with NWTSG was $1.6 \%$ and increasing. Further study is required to validate these facts.

In 2001, Tournade et al. (5) reported that preoperative chemotherapy with vincristine and dactinomycin for four weeks was as effective as for eight weeks in localized Wilms' tumor. According to this conclusion, short (2 - 4 weeks) course of preoperative chemotherapy was proposed, which reduces the outcome of therapeutic toxicity. The pooled RR for TS, TR, EFS in our study respectively are 1.097 (95\% CI: $0.784-1.563 ; \mathrm{P}=0.587 ; \mathrm{I}^{2}=0 \%$ ), 1.197 (95\% CI: $0.960-1.493 ; \mathrm{P}=0.110 ; \mathrm{I}^{2}=0 \%$ ) and 1.006 (95\% CI: 0.910 $-1.250 ; P=0.430 ; I^{2}=0 \%$ ) for subgroup SC vs. LC. Though there is a tendency to increase TS and TR compared to LC group, the differences are not statistically significant. The short course therapy was as effective as conventional intravenous chemotherapy (4- 6 weeks duration, SIOP recommends). Also, our results are intact with the SIOP (9). The mechanism may be associated with the drug-resistance, which is associated with multidrug resistanceassociated protein (MRP). It has been shown that the tumor shrinks remarkably during second-third week of preoperative chemotherapy while after the fourth week there could be a tendency of the tumor size to increase because of the MRP expression.

There are also some limitations in our meta-analysis: the studies included are 19 English and 13 Chinese, publication bias could not be ignored; the studies included are not all RCTs, the retrospective studies also cause bias in methodology; histological characteristics and stage levels also play an important role in the prognosis of Wilm' tumor but we did not include these factors in the study. Apart from these limitations, we carried out the first meta-analysis on preoperative chemotherapy and its subgroup with the largest patient number.

In summary, our meta-analysis shows preoperative chemotherapy benefits Wilms' tumor patients in tumor shrinkage and resection but not event-free survival. Meanwhile, the efficacy of short course chemotherapy is similar to long course chemotherapy. In other words, short course chemotherapy can be offered as an effective and safe alternative treatment modality for Wilms' tumor. But the patients included in SC groups are few; further study with more RCTs and large scale patients are needed to overcome the limitations that existed in this study.

\section{References}

1. SIOP PODC: clinical guidelines for the management of children with Wilms tumour in a low income setting. Israels T, Moreira C, Scanlan T, Molyneux L, Kampondeni S, Hesseling P, et al. Pediatr Blood Cancer. 2013;60(1):5.

2. Epidemiology of Wilms tumor. Breslow N, Olshan A, Beckwith JB, Green DM. Med Pediatr Oncol. 1993;21(3):172.

3. Treatment and outcome of Wilms' tumour patients: an analysis of all cases registered in the UKW3 trial. Pritchard-Jones K, Moroz V, Vujanic G, Powis M, Walker J, Messahel B, et al. Ann Oncol. 2012;23(9):2457.
4. Preoperative regimen and surgery time in children Wilms' tumor. He XB, Liu GQ, Li CC. ShiYong Yi Xue Za Zhi. 20 05;21(12):1289.

5. Optimal duration of preoperative therapy in unilateral and nonmetastatic Wilms' tumor in children older than 6 months: results of the Ninth International Society of Pediatric Oncology Wilms' Tumor Trial and Study. Tournade MF, Com-Nougue C, de Kraker J, Ludwig R, Rey A, Burgers JM, et al. J Clin Oncol. 2001;19(2):488.

6. Higgins JPT, Green S. Cochrane Handbook for Systematic Reviews of Interventions. Chichester: Wiley; 2008.

7. New guidelines to evaluate the response to treatment in solid tumors. European Organization for Research and Treatment of Cancer, National Cancer Institute of the United States, National Cancer Institute of Canada. Therasse P, Arbuck SG, Eisenhauer EA, Wanders J, Kaplan RS, Rubinstein L, et al. J Natl Cancer Inst. 2000;92(3):205.

8. Meta-analysis in clinical trials. DerSimonian R, Laird N. Control Clin Trials. 1986;7(3):177.

9. Assessing heterogeneity in meta-analysis: Q statistic or I2 index? Huedo-Medina TB, Sanchez-Meca J, Marin-Martinez F, Botella J. Psychol Methods. 2006;11(2):193.

10. Operating characteristics of a rank correlation test for publication bias. Begg CB, Mazumdar M. Biometrics. 1994;50(4):1088.

11. Evaluation of preoperative chemotherapy project and selection of operative time of nephroblastoma in children. Liu GQ, Tang HJ, Wang JF. Zhong Hua Xian Dai Er Ke Za Zhi. 2004;1(2):112.

12. The analysis of preoperative chemotherapy in late stage patients with wilms' tumor. Hang YC, Han GS, Ren JK. Zhong Guo Mei Tan Gong Ye Yi Xue Za Zhi. 2006;9(9):950.

13. Clinical analysis of preoperative chemotherapy in patients with wilms' tumor. Sun J, Yi J. Nan Jing Yi Ke Da Xue Xue Bao. 1997;17(3):275.

14. The influence of preoperative chemotherapy in children with wilms' tumor. Lu XZ, Wang AX, Zuo WL, Guan YB. Ren Min Jun Yi. 2008;10(1):56.

15. The pathology changes after preoperative chemotherapy in patients with wilms' tumor. Gao JC, Chen L. Zhong Hua Mi NiaoWai Ke Za Zhi. 1996;17(11):654.

16. Preoperative chemotherapy in late stage patients with wilms' tumor. Huang Y, Li MJ, Zhou YB. Zhong Hua Xiao Er Wai Ke Za Zhi. 2004;25(3):205.

17. Intratumoral chemotherapy in wilms' tumor. Li JP, Guo WB. Zhong Hua Xiao Er Wai Ke Za Zhi. 2002;23(3):205.

18. The 57 cases of wilms' tumor. Tang HJ, Liu GQ, Wang JF. Zhong Guo Yi Shi Za Zhi. 2005;7(4):544.

19. A retrospective study of the preoperative treatment of advanced Wilms tumor in children with chemotherapy versus transcatheter arterial chemoembolization alone or combined with shortterm systemic chemotherapy. Li MJ, Zhou YB, Huang Y, Tang DX, Xu S, Wu DH, et al. J Vasc Interv Radiol. 2011;22(3):279.

20. The efficacy of preoperative chemotherapy in wilms' tumor. Zhao YB, Zheng SB, Mao XM. Di Yi Jun Yi Da Xue Xue Bao. 2004;24(6):722.

21. The advantages of preoperative chemotherapy in patients with wilms' tumor. Tang DX, LI MJ. ShiYong Zhong Liu Za Zhi. 2001;16(1):39.

22. Treatment of advanced wilms' tumor. Li MJ, Huang Y, Tang DX. Zhong Hua Zhong Liu Za Zhi. 2006;28(10):791.

23. Multidisciplinary approach to wilms' tumor: a retrospective analytical study of 53 patients. Naguib SF, El Haddad A, El Badawy SA, Zaghloul AS. J Egypt Natl Canc Inst. 2008;20(4):410.

24. Multidisciplinary approach to Wilms' tumor: 18 years of experience. Yildiz I, Yuksel L, Ozkan A, Apak H, Celkan T, Danismend N, et al. Jpn J Clin Oncol. 2000;30(1):17.

25. Bilateral Wilms' tumors: single-center experience with 22 cases and literature review. Sarhan OM, El-Baz M, Sarhan MM, Ghali AM, Ghoneim MA. Urology. 2010;76(4):946.

26. From upfront nephrectomy to preoperative chemotherapy and back: a single institution experience in the treatment of Wilms tumor. Sultan I, Masarweh M, Ismael T, Al-Hussaini M, Almousa A, Ali HM, et al. J Pediatr Hematol Oncol. 2009;31(5):333.

27. Does preoperative chemotherapy ease the surgical procedure 
for Wilms tumor? Bogaert GA, Heremans B, Renard M, Bruninx L, De Wever L, Van Poppel H. J Urol. 2009;182(4 Suppl):1869.

28. Outcome of children with cystic partially differentiated nephroblastoma treated with or without chemotherapy. Blakely ML, Shamberger RC, Norkool P, Beckwith JB, Green DM, Ritchey ML, et al. J Pediatr Surg. 2003;38(6):897.

29. Intracaval and intracardiac extension of Wilms' tumor. The influence of preoperative chemotherapy on surgical morbidity. Cristofani LM, Duarte RJ, Almeida MT, Odone Filho V, Maksoud IG, Srougi M. Int Braz J Urol. 2007:33(5):683. discussion 689.

30. Childhood renal tumours with intravascular extension. Mushtaq I, Carachi R, Roy G, Azmy A. Br J Urol. 1996;78(5):772.

31. Conservative surgical management of bilateral Wilms tumor: results of the United Kingdom Children's Cancer Study Group. Kumar R, Fitzgerald R, Breatnach F. J Urol. 1998;160(4):1450.

32. Fetal rhabdomyomatous nephroblastoma: a tumour of good prognosis but resistant to chemotherapy. Maes P, Delemarre J, de Kraker J, Ninane J. Eur J Cancer. 1999;35(9):1356.

33. Treatment of bilateral Wilms' tumor: comparison of initial biopsy and chemotherapy to initial surgical resection in the preservation of renal mass and function. Shaul DB, Srikanth MM, Ortega JA, Mahour GH. J Pediatr Surg. 1992;27(8):1009. discussion 1014-5.

34. Clinical impact of histologic subtypes in localized non-anaplastic nephroblastoma treated according to the trial and study SIOP-9/GPOH. Weirich A, Leuschner I, Harms D, Vujanic GM, Troger J, Abel U, et al. Ann Oncol. 2001;12(3):311.

35. Preoperative care of infants with nephroblastoma. The International Society of Pediatric Oncology 6 experience. Coppes MJ, Tournade MF, Lemerle J, Weitzman S, Rey A, Burger D, et al. Cancer. 1992;69(11):2721.

36. Chemotherapy-induced histologic changes in Wilms' tumors. Guarda LA, Ayala AG, Jaffe N, Sutow WW, Bracken RB. Pediatr Pathol.1984;2(2):197.

37. Wilms' tumour: a comparison of surgical aspects in patients with or without pre-operative chemotherapy. Safdar CA, Aslam
M, Awan SH, Ahmed I, Badshah S. J Coll Physicians Surg Pak. 2006;16(8):521.

38. Preoperative versus postoperative radiotherapy, single versus multiple courses of actinomycin D, in the treatment of Wilms' tumor. Preliminary results of a controlled clinical trial conducted by the International Society of Paediatric Oncology (S.I.O.P.). Lemerle J, Voute PA, Tournade MF, Delemarre JF, Jereb B, Ahstrom L, et al. Cancer. 1976;38(2):647.

39. Immediate nephrectomy versus preoperative chemotherapy in the management of non-metastatic Wilms' tumour: results of a randomised trial (UKW3) by the UK Children's Cancer Study Group. Mitchell C, Pritchard-Jones K, Shannon R, Hutton C, Stevens S, Machin D, et al. Eur J Cancer. 2006;42(15):2554 .

40. The treatment of Wilms' tumor: Results of the national Wilms' tumor study. D'Angio GJ, Evans AE, Breslow N, Beckwith B, Bishop H, Feigl P, et al. Cancer. 1976;38(2):633.

41. Wilms' tumor and other renal tumors of childhood: a selective review from the National Wilms' Tumor Study Pathology Center. Beckwith JB. Hum Pathol. 1983;14(6):481.

42. Complete necrosis induced by preoperative chemotherapy in Wilms tumor as an indicator of low risk: report of the international society of paediatric oncology (SIOP) nephroblastoma trial and study 9. Boccon-Gibod L, Rey A, Sandstedt B, Delemarre J, Harms D, Vujanic G, et al. Med Pediatr Oncol. 2000;34(3):183.

43. Reduction of postoperative chemotherapy in children with stage I intermediate-risk and anaplastic Wilms' tumour (SIOP 93-01 trial): a randomised controlled trial. de Kraker J, Graf N, van Tinteren H, Pein F, Sandstedt B, Godzinski J, et al. Lancet. 2004;364(9441):1229.

44. The efficacy and toxicity of SIOP preoperative chemotherapy in Malawian children with a Wilms tumour. Israels T, Chagaluka G, Pidini D, Caron H, de Kraker J, Kamiza S, et al. Pediatr Blood Cancer. 2012;59(4):636.

45. The expression of p-glycoprotein in Wilms' tumor. Wei GH, Jin XQ. Chong Qing Yi Ke Da Xue Xue Bao. 2001;26(1):34. 